Regina Widmer

\section{Mut machen}

Dieser Artikel soll den interessierten Fachleuten Mut machen, ihren Weg im unübersichtlichen Angebot von konfektionierten und von Apothekerhand hergestellten Phytotherapeutika für den Intimbereich zu finden. Es gibt nur wenige bis keine klinischen Studien dazu.

Ich bin glücklich über das Editorial. Wir haben als Medizinerinnen und Mediziner in unserer Ausbildung nichts gelernt über Phytotherapie. Die Nachfrage der Frauen nach empfehlenswerten Produkten ist gross - die ärztliche Hilflosigkeit auch. Mangels praxistauglicher Zusammenstellungen, Eigenerfahrung und Zeitnot greifen wir oft zu den immer gleichen Produkten, denen wir während der klinischen Ausbildung begegnet sind. Und von den in der Praxis gemachten ausgezeichneten Erfahrungen mit phytotherapeutischen Präparaten und Magistralrezepturen fliesst praktisch nichts zurück in die Klinik.

\section{Pflanzliche Gynäkologika}

Die Vorliebe von Frauen für natürliche Gynäkologika ist an den Pharmafirmen nicht vorbeigegangen. Erfreulicherweise lässt sich eine wachsende Zahl an recht guten phytotherapeutischen Produkten für die Intimpflege finden, über die es leider keine Übersicht gibt. Häufig fallen sie unter Medizinprodukte, einige unter Kosmetika. Eine grosse Zahl an pflanzlichen

\section{Einsatz von Phytotherapeutika bei Vulvovaginalbeschwerden}

Kasten 1. Umwidmen von Präparaten für den gynäkologischen Gebrauch

\begin{tabular}{rl|}
$\Rightarrow$ & Viele hochwertige phytotherapeutische Prä- \\
& parate für die Gesichts- und Babypflege eig- \\
& nen sich hervorragend für die Vulva, z.B. \\
& Calendula und Weisse Malve Babycrème ${ }^{\circledR}$ \\
$\Rightarrow$ & Phytotherapeutische Suppositorien, wie Ha- \\
& metum $^{\circledR}$, können auch vaginal angewandt \\
& werden (Gerbstoffe, bei Kontaktblutungen) \\
$\Rightarrow$ & Phytotherapeutischer beruhigender Nasen- \\
& ${\text { spray Yerbasin Nose }{ }^{\circledR} \text { für Vorhof, Introitus }}$
\end{tabular}

Fertigpräparaten für den Intimbereich findet sich als anthroposophische Heilmittel. Der Einfachheit halber subsumiere ich diese unter Phytotherapeutika.

Manchmal ist es am einfachsten und schnellsten, ein konfektioniertes Fertigprodukt anzuwenden. Es empfiehlt sich, einen Grundstock anzulegen oder verordnen zu können (Kasten 1, 2).

\section{Von der Apothekerin selbst hergestellte Phytotherapeutika}

Die Hürde, sich Zugang zu hochqualitativen, in kleiner Produktion hergestellten Intimica zu verschaffen, ist nicht so hoch, wie manche denken (Kasten 2, 3). Suchen Sie sich eine Apothekerin in Ihrer Umgebung, wählen Sie eine der aufgelisteten Referenzapotheken der SMGP (Schweizerische Medizinische Gesellschaft für Phytotherapie, smgp.ch) oder besuchen Sie eine der in Kasten 4 angegebenen Homepages und nehmen Sie persönlich Kontakt mit der verantwortlichen Apothekerin auf.
Kasten 2. Phytotherapeutika für Anfänger und Fortgeschrittene

Für Anfänger
$\Rightarrow$ Entscheiden Sie sich für einige wenige phyto-
therapeutische Fertigprodukte und sammeln
Sie Erfahrungen damit; z.B. ein feuchtigkeit-
spendendes Ovulum, ein phytoöstrogenhalti-
ges Ovulum, eine hautnährende Crème oder
ein Öl für die Vulva, eine Crème für Vulva
und Vagina, eine schützende Crème für vul-
nerable Vulven.
$\Rightarrow$ Suchen Sie sich eine Apothekerin in Ihrer
Nähe oder online, die sich in phytotherapeu-
tischen Magistralrezepturen auskennt und/
oder gynäkologische Hausspezialitäten her-
stellt. Sie müssen nicht selber alles wissen.
Testen Sie ein, zwei handgemachte Präparate
(am besten an sich selber).
$\Rightarrow$ Bilden Sie sich weiter, z.B. in Kursen der
SMGP, Herbadonna usw.
Für Fortgeschrittene
$\Rightarrow$ Erweitern Sie Ihr Sortiment/Ihre Empfehlun-
gen. Tauschen Sie sich unter Fachleuten aus
und geben Sie Ihre Erfahrung weiter.

Kasten 3. Informationsmöglichkeiten

$\Rightarrow$ Herbadonna (herbadonna.ch)

$\Rightarrow$ Netzwerk gynäkologisch-phytotherapeutisch interessierter Fachleute mit Workshops in Solothurn und Zürich, der SMGP (smgp.ch) angegliedert

Kasten 4. Auswahl empfehlenswerter Homepages mit phytotherapeutischen Intimica

$\Rightarrow$ zentral-apo.ch, Petra Hagel
$\Rightarrow$ femisanum.com, Patrizia Ochsner
$\Rightarrow$ medizinfeminin.ch, Dorin Ritzmann
$\Rightarrow$ berg-apotheke.ch, Nelly Richina
$\Rightarrow$ saner-apotheke.ch, Susanne Villalonga
$\Rightarrow$ frauenpraxis-runa.ch, Merkblätter, Therapie-
$\quad$ empfehlungen für den Intimbereich

\section{KARGER}

๑) 2017 S. Karger GmbH, Freiburg

Fax +497614520714 
Hochqualitativ heisst: hochwertige Inhaltsstoffe (beste ätherische Öle, Extrakte usw.), aber auch hochwertige Salben- und Ovulagrundlagen. Darüber haben wir ebenfalls nichts gelernt in unserer medizinischen Ausbildung. Hierzu folgende Empfehlung: Mineralöle und ihre Abkömmlinge haben nichts in Pflegeprodukten verloren! Also Hände weg bei Inhaltsstoffen wie Paraffinum liquidum, Vaselinum album usw. Den Rest wissen die pharmazeutischen Kolleginnen und Kollegen, nämlich was befeuchtet, schützt, trocknet, klebt oder fettet. Idealerweise soll die Trägersubstanz nicht nur Füllmaterial sein, sondern gleichzeitig auch einen therapeutischen Effekt erzielen. So haben Vaginal-Ovula - notabene alle konfektionierten, auch die phytotherapeutischen - ein neutrales Hartfett als Grundlage. Bei einer Magistralrezeptur hingegen kann nicht nur der Wirkstoff, sondern auch die Grundlage gewählt werden, wie z.B. Kakaobutter, welche selber eine intimpflegende Wirkung hat. Es empfiehlt sich, ein paar Rezepturen zu kennen.

Selbst praktiziere ich als Gynäkologin seit 24 Jahren in eigener Gruppenpraxis (20 Jahre Frauenpraxis Runa in Solothurn, davor 4 Jahre Frauenambulatorium im Frauenzentrum Zürich, dazwischen klinische Jahre).

\section{Wie vorgehen bei Vulvovaginalbeschwerden?}

- In der Klinik ist es kaum möglich, Frauen mit chronischen Vulvovaginalbeschwerden zu begleiten, am ehesten noch in einer gynäkologischen Spezialsprechstunde oder was noch seltener existiert - einer dermatologisch-gynäkologischen Sprechstunde. So begegnen wir dem wirklichen Ausmass an alltäglichen störenden Beschwerden in der Praxis. Dort können wir uns all den kleinen «Rissli» und überempfindlichen Hautstellen der Vulva widmen. Über die Jahre habe ich die meisten Therapieerfolge erlebt, wenn ich mich $100 \%$ für die «kleinen Bobos» interessiere, die traditionell in unserer Fachausbildung zu den lästigeren «Musts» gehören. Der Schreck der Gynäkologinnen und Gynäkologen: Die Patientin hat Beschwerden wie Vulvodynie/ Vestibulitis und die Gynäkologin bzw. der Gynäkologe «sieht nichts».

- Daher sollte eine genaue Anamnese mit Beginn der Beschwerden erfolgen. Hilfreich ist ein von der Frau zusammengestelltes chronologisches "Curriculum der Beschwerden». Dann folgt eine gründliche gemeinsame Untersuchung - «Wo genau tut es weh?»-, bis anhand oft diffuser lokaler Angaben die Stellen gefunden sind. Gemeinsam heisst: mit Handspiegel, den die Frau hält. Die Patientin zeigt mit dem Finger die Beschwerdestelle so genau wie möglich. Die Untersucherin bzw. der Untersucher inspiziert und palpiert selbst genau. Palpatorisch sollte das untere Vaginaldrittel mit einbezogen werden - vor allem, wenn der Verdacht auf eine schmerzhafte hypertone Beckenbodenmuskulatur besteht.

- Sehr oft erweisen sich von der Frau empfundene vaginale Beschwerden als solche vulvärer Art. Dies ist schon fast die Regel bei beklagten Penetrationsbeschwerden, welche die Frau «innen» empfindet, die sich aber wie die meisten Beschwerden am Introitus (Scheideneingang) und im Vestibulum (Scheidenvorhof) befinden.

- Ist es ein Hautproblem? Handelt es sich um ein Muskelproblem (hypertone Beckenbodenmuskulatur)? Oder um beides?

- Selbstverständlich gehört die vaginale Untersuchung mit dem Spekulum dazu - mit folgenden Fragen: Gut epithelialisierte Vagina? Feucht? Vaginalinhalt physiologisch? $\mathrm{KOH}-$ Probe und Nativpräparat.

- Es bewähren sich die «5 $A$ » für Vulva + Vagina (+ Anus) (Kasten 5). Darauf basierend ist eine Hierar-
Kasten 5. Es bewähren sich die «5 A» für Vulva + Vagina (+ Anus), nach Regina Widmer

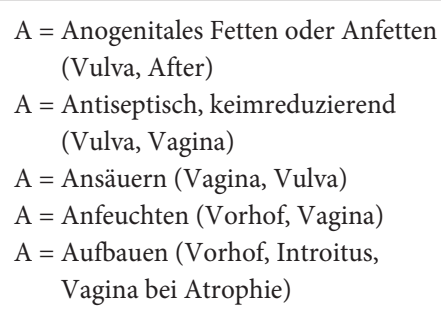

$\mathrm{A}=$ Anogenitales Fetten oder Anfetten (Vulva, After)

$\mathrm{A}=$ Antiseptisch, keimreduzierend (Vulva, Vagina)

$\mathrm{A}=$ Ansäuern (Vagina, Vulva)

$\mathrm{A}=$ Anfeuchten (Vorhof, Vagina)

$\mathrm{A}=$ Aufbauen (Vorhof, Introitus,

Vagina bei Atrophie)

chie zu erstellen mit den zwei prioritären Beschwerden bzw. Therapien und eine Entscheidung für entsprechende Produkte wird getroffen. Meine Präferenz sind hochwertige phytotherapeutische Produkte, begleitend oder im Hintergrund durchaus auch synthetische unersetzliche Präparate.

- So erhalten bei mir alle Frauen mit Lichen sclerosus eine phytotherapeutische tägliche Grundpflege und haben im Hintergrund eine CortisonCrème für akute Schübe zur Hand.

- Alle Frauen mit atrophen Vaginalschleimhäuten erhalten von mir eine phytotherapeutische tägliche Grundpflege und ein sporadisch anzuwendendes synthetisches Östrogen - beides als Crème und/oder Ovula.

- Bei Dysplasien der Portio kommen gerbstoffhaltige Ovula infrage.

- Wenn wir uns um die vulvovaginale Gesundheit bzw. Gesundung kümmern, hat dies eine weitreichende Wirkung auf die Frau, allem voran auf ihr sexuelles Wohlbefinden und damit auf ihr allgemeines Wohlbefinden.

- Tägliche Intimpflege fördert den positiven Bezug der Frau zu ihren Genitalien und ihrer Intimität.

- Frauen mit heiklen Vulven, die beim Geschlechtsverkehr leiden, sollen nicht nur auserlesene Gleitmittel benutzen, sondern eine tägliche Intimpflege installieren. Die Haut und Schleimhäute sollen grundsätzlich genährter, dicker, feuchter und resistenter werden. Wenn zusätzlich Gleitmittel benötigt werden, dann profitiert die Intimhaut von Frau und Mann zu- 
Kasten 6. Wie vorgehen bei vulvovaginalen Beschwerden?

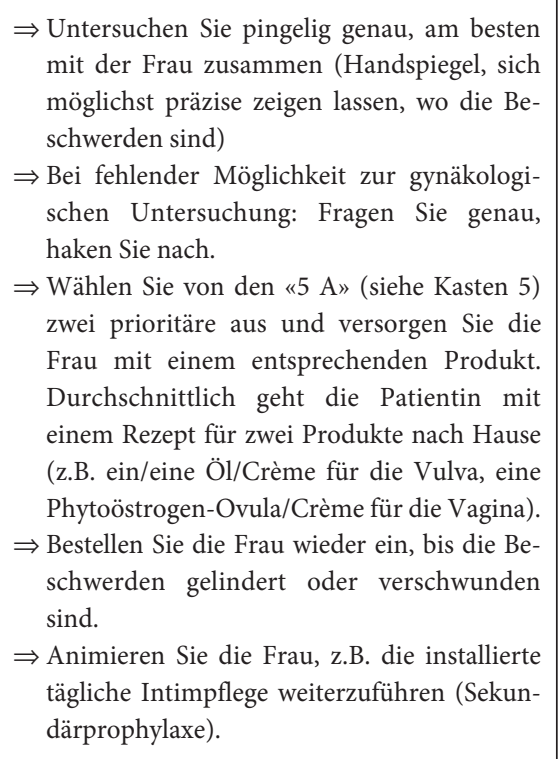

sätzlich von pflanzlichem Gleitöl (nicht mit Kondom) wie z.B. einem neutralen Mandelöl oder einem duftenden Granatapfelöl (Abb. 1). Bei sehr heiklen Häuten und lange andauerndem Liebesspiel geht nichts über Silicon-Gleitmittel. Diese legen sich schützend auf die Haut, werden nicht resorbiert und gleiten hervorragend, ohne matschig zu werden.

- Es gehört immer noch nicht zum Allgemeinwissen der Frauen, dass sie ihre Intimhaut pflegen können und auch selbst ausprobieren dürfen, was sie vertragen oder nicht. 1-2 Mittel als Empfehlung für den Beginn ist sinnvoll.

\section{Einblick in meine konkrete phytotherapeutische Handhabung der gängigsten Vulvovaginalbeschwerden}

Dies ist meine persönliche Hitliste und soll nur als Beispiel dienen und sozusagen die Machbarkeit illustrieren (Kasten 6). Tagtäglich verwende ich nur eine kleine Auswahl an Phytotherapeutika. Es gibt allerdings andere ebenso gute Heilmittel. 80\% der gynä- kologischen Beschwerden behandle ich phytotherapeutisch. Manchmal kombiniere ich die Phytotherapeutika mit synthetischen Medikamenten.

- Kondylome der Vulva: GrünteeExtrakt (Veregen ${ }^{\circledR}$ ) (Abb. 2).

- Dysplasien der Portio: Eiben-Ovula nach Dorin Ritzmann (siehe medizinfeminin.ch).

- Blutende Ektopien der Portio (Kontaktblutungen): Gerbstoffe, entweder Argentum/Quercus comp. ${ }^{\circledR} \mathrm{Va}$ ginal Globuli oder stärker gerbstoffhaltige Magistralrezeptur für Eichenrinden-Ovula (diverse Phytoreferenzapotheken).

- Hartnäckige Rhagaden Vulva/ Anus: Hamamelis Quercus comp. ${ }^{\circledR}$ mit Zink = haftet gut an feuchte Vulva/Vestibulum.

- Bartholin-Zysten/Atherome/beginnende Abszedierungen der Vulva: Fichtenharzbalsam als pflanzliche Zugsalbe (z.B. Berg-Apotheke Zürich).

- Atrophien der Vagina und Vulva: Rheum rhaponticum D6 Crème oder Ovula (Abb. 3) oder Magistralrezeptur vaginale Kräutercrème (wird von etlichen Apotheken hergestellt).

- Affinität der Vulva für Pilzinfektionen: Lavendelöl (ätherisches Öl von Lavendel in Weizenkeim- und Mandelöl) als Sekundärprophylaxe und als tägliche Grundpflege.

- Lichen sclerosus: Lavendelöl täglich.

- Bei entzündlichen Vulven und offenen Stellen: Weisse Malve Babycrème $^{\circledR}$, Calendula Babycrème ${ }^{\circledR}$ (beide zinkhaltig), Remederm Silber Crème ${ }^{\circledR}$.

- Bei heikler Vulvahaut: Cobagin ${ }^{\circledR}$, Lavendöl verdünnt als Magistralrezeptur.

- Vaginale Pilzinfektion: TeebaumOvula 5\% (Magistralrezeptur).

- Bakterielle Vaginose: Ansäuerung.

- Streptokokken-B-Besiedelung bei Schwangeren: Lavendel-Ovula 1\% im letzten Trimenon.

- Bei unspezifischen Kolpitiden: Majorana/Melissa ${ }^{\circledR}$ Vaginaltabletten, Majorana Vaginal-Gel ${ }^{\circledR}$.

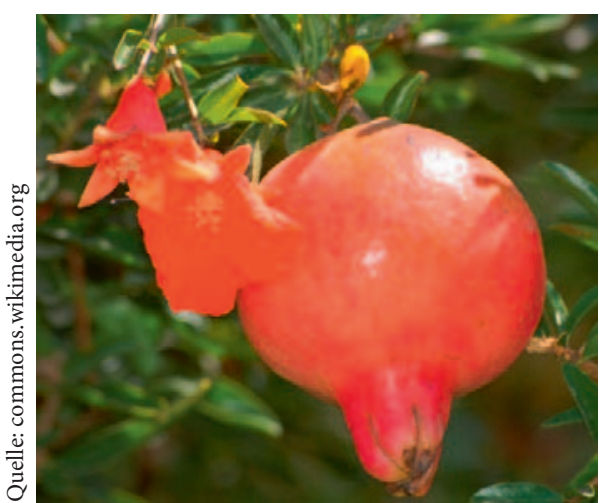

Abb. 1. Granatapfel (Punica granatum).

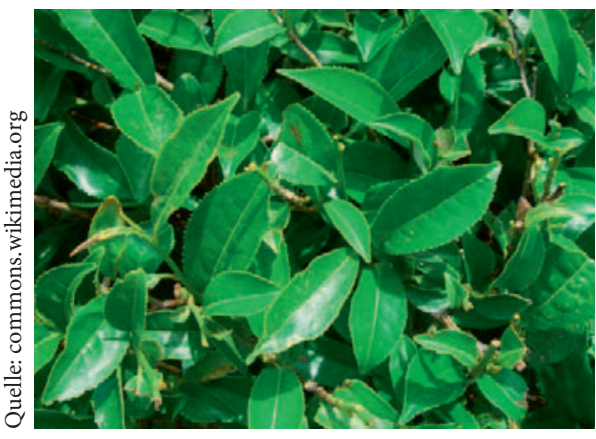

Abb. 2. Grüntee (Camellia sinsensis).

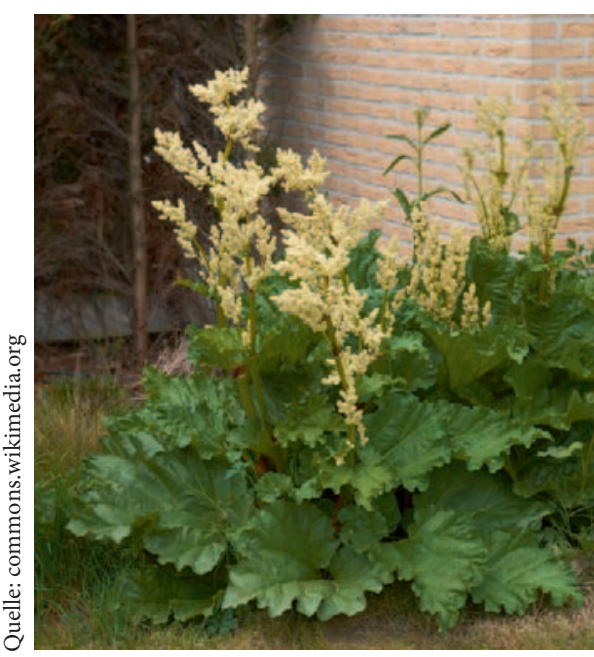

Abb. 3. Sibirischer Rhabarber (Rheum rhaponticum).

\section{Weitere nützliche Links,} Adressen, Bücher:

- Alle Bücher von Heide Fischer und Margret Madejsky.

- Phyto-Kompendium von Beatrix Falch (phytocura.ch).

- «Natürlich durch die Wechseljahre» von Ruth Jahn und Regina Widmer, Beobachter-Ratgeber. 